\title{
BIBASIC $q$-APPELL SERIES CONNECTED WITH RAMANUJAN'S CONTINUED FRACTIONS
}

\author{
BHASKAR SRIVASTAVA
}

\begin{abstract}
In this paper we give some summation formulae for bibasic $q$-Appell function $\bar{\varphi}^{(1)}$ and express Appell function $\bar{\varphi}^{(1)}$ in terms of classical continued fractions of Ramanujan. We also express the $q$-Appell function $\varphi^{(1)}$ on one base as a continued fraction. The bibasic approach for the Appell series, we think, is new.
\end{abstract}

\section{Introduction}

The basic analogues of Appell's hypergeometric functions of two variables were first studied and defined by F. H. Jackson 7, 8]. Much is known about ordinary Appell series. However, not so much is known about basic Appell series. Slater [9, p.234] remarked that no systematic attempt has been made to find summation theorems for basic Appell series. Slater has applied contour integral method to these series.

We have taken a new approach, by defining and considering bibasic Appell series and we published the first paper Srivastava [10] on the subject. Now we have made a comprehensive study of the bibasic Appell series.

In this paper we have given summation formulae and continued fraction representation for these bibasic Appell functions. These continued fractions are those given by Ramanujan. Thus we have given a relation between bibasic Appell series and continued fractions of Ramanujan. We think these results are not found in the literature.

\section{Background}

The first basic Appell series defined by Jackson is

$$
\varphi^{(1)}\left[a ; b, b^{\prime} ; c ; x, y\right]=\sum_{m=0}^{\infty} \sum_{n=0}^{\infty} \frac{(a)_{m+n}(b)_{m}\left(b^{\prime}\right)_{n}}{(q)_{m}(q)_{n}(c)_{m+n}} x^{m} y^{n} .
$$

We defined bibasic on two independent bases $q, q_{1},|q|,\left|q_{1}\right|<1$ as

$$
\bar{\varphi}^{(1)}\left[a ; b, b^{\prime} ; c ; x, y ; q, q_{1}\right]=\sum_{m=0}^{\infty} \sum_{n=0}^{\infty} \frac{\left(a ; q_{1}\right)_{m+n}(b ; q)_{m}\left(b^{\prime} ; q\right)_{n}}{(q ; q)_{m}(q ; q)_{n}\left(c ; q_{1}\right)_{m+n}} x^{m} y^{n} .
$$

Received August 16, 2005.

2000 Mathematics Subject Classification. 33D65, 33C65.

Key words and phrases. $q$-bibasic hypergeometric series, $q$-Appell, continued fraction. 


\section{Notation}

The following $q$-notation is used: For $\left|q^{k}\right|<1$

$$
\begin{aligned}
& \left(a ; q^{k}\right)_{n}=(1-a)\left(1-a q^{k}\right) \cdots\left(1-a q^{k(n-1)}\right), \quad n \geq 1 \\
& \left(a ; q^{k}\right)_{0}=1 \\
& \left(a ; q^{k}\right)_{\infty}=\prod_{j=0}^{\infty}\left(1-a q^{k j}\right), \\
& \left(a_{1}, a_{2}, \ldots, a_{m} ; q^{k}\right)_{n}=\left(a_{1} ; q^{k}\right)_{n}\left(a_{2} ; q^{k}\right)_{n} \cdots\left(a_{m} ; q^{k}\right)_{n}, \\
& (a)_{n}=(a ; q)_{n}=(1-a)(1-a q) \cdots\left(1-a q^{n-1}\right) .
\end{aligned}
$$

A generalized basic hypergeometric series with base $q_{1}$ is defined as ${ }_{A} \phi_{A-1}\left[a_{1}, a_{2}, \ldots, a_{A} ; b_{1}, b_{2}, \ldots, b_{A-1} ; q_{1}, z\right]=\sum_{n=0}^{\infty} \frac{\left(a_{1} ; q_{1}\right)_{n} \cdots\left(a_{A} ; q_{1}\right)_{n} z^{n}}{\left(b_{1} ; q_{1}\right)_{n} \cdots\left(b_{A-1} ; q_{1}\right)_{n}\left(q ; q_{1}\right)_{n}},|z|<1$.

\section{Continued fraction for $\bar{\varphi}^{(1)}$}

In this section we will give a continued fraction representation for the bibasic Appell function $\bar{\varphi}^{(1)}$.

Theorem 1.

$$
\begin{aligned}
\frac{\bar{\varphi}^{(1)}\left[a ; b, \frac{x}{y} ; b^{2} b^{\prime 2} ; x, y ; q, q^{2}\right]}{\bar{\varphi}^{(1)}\left[a q^{2} ; b q^{2}, \frac{x}{y} ; b^{2} b^{\prime 2} q^{4} ; x, y ; q, q^{2}\right]} \\
=\frac{T}{S}+\frac{U / S}{\frac{\varphi^{(1)}\left[a q^{2} ; b q^{2}, \frac{x}{y} ; b^{2} b^{\prime 2} q^{4} ; x, y ; q, q^{2}\right]}{\varphi^{(1)}\left[a q^{4} ; b q^{4}, \frac{x}{y} ; b^{2} b^{\prime 2} q^{8} ; x, y ; q, q^{2}\right]}}+\cdots
\end{aligned}
$$

$S, T$ and $U$ are defined in the proof below.

Proof. We have proved in Theorem 1 [10, p.31] that

$$
\begin{aligned}
& \bar{\varphi}^{(1)}\left[a ; b, b^{\prime}, c ; x, y ; q ; q^{k}\right]=\frac{\left(a ; q^{k}\right)_{\infty}(b x ; q)_{\infty}\left(b^{\prime} y ; q\right)_{\infty}}{\left(c ; q^{k}\right)_{\infty}(x ; q)_{\infty}(y ; q)_{\infty}} \\
& \times_{2 k+1} \phi_{2 k}\left[\begin{array}{l}
c / a, x, x q, x q^{2}, \ldots, x q^{k-1}, y, y q, y q^{2}, \ldots, y q^{k-1} \\
b x, b x q, \ldots, b x q^{k-1}, b^{\prime} y, b^{\prime} y q, \ldots, b^{\prime} y q^{k-1}
\end{array} ; q^{k}, a\right] .
\end{aligned}
$$

By takinig $k=1$, we have the Theorem 1 of Andrews [1, p.619]. 
Putting $k=2, b^{\prime}=\frac{x}{y}, c=b^{2} b^{\prime 2}$ in (4.1), we have

$$
\begin{aligned}
\bar{\varphi}^{(1)}\left[a ; b, \frac{x}{y} ; b^{2} b^{\prime 2} ; x, y ; q ; q^{2}\right]= & \frac{\left(a ; q^{2}\right)_{\infty}\left(b b^{\prime} y ; q^{2}\right)_{\infty}}{(y ; q)_{\infty}\left(b^{2} b^{\prime 2} ; q^{2}\right)_{\infty}} \\
& \times{ }_{3} \phi_{2}\left(\frac{b^{2} b^{\prime 2}}{a}, y, y q ; b b^{\prime} y, b b^{\prime} y q ; q^{2}, a\right) .
\end{aligned}
$$

Using the contiguous relation of Andrews and Bowman [3, p.3347, (3.13)], we have

$$
\begin{aligned}
S \bar{\varphi}^{(1)}\left[a ; b, \frac{x}{y} ; b^{2} b^{\prime 2} ; x, y ; q, q^{2}\right]= & T \bar{\varphi}^{(1)}\left[a q^{2} ; b q, \frac{x}{y}, b^{2} b^{\prime 2} q^{4} ; x, y ; q, q^{2}\right] \\
& +U \bar{\varphi}^{(1)}\left[a q^{4} ; b q^{2}, \frac{x}{y}, b^{2} b^{\prime 2} q^{8} ; x, y ; q, q^{2}\right],
\end{aligned}
$$

where

$$
\begin{aligned}
& S=\frac{b^{2} b^{\prime 2} y^{6} q^{3}}{a}\left(1-a q^{2}\right)\left(1-b^{2} b^{\prime 2} q^{4}\right)\left(1-b^{2} b^{\prime 2}\right)\left(b^{2} b^{\prime 2} q^{2} ; q^{2}\right)_{4} \\
& T=b^{2} b^{\prime 2} y^{6} q^{3}\left(1-a q^{2}\right)\left(b^{2} b^{\prime 2} q^{2} ; q^{2}\right)_{2}\left[-y\left(1+b b^{\prime} / a\right)\left(b b^{\prime} ; q^{2}\right)(1+q)+\frac{1}{a}\left(b^{2} b^{\prime 2} ; q^{4}\right)_{2}\right] \\
& U=b^{2} b^{\prime 2} y^{8} q^{4}\left(1+b b^{\prime}\right)\left(1-b b^{\prime} q^{2}\right)\left(1-b^{2} b^{\prime 2} q^{2} / a\right)\left(b b^{\prime} ; q\right)_{4}\left(b^{2} b^{\prime 2} q^{8} ; q^{2}\right)_{4} .
\end{aligned}
$$

Iterating the contiguous relation (4.3), we have the Theorem.

\section{Continued fraction for $\varphi^{(1)}$}

\section{Theorem 2.}

$$
\begin{aligned}
& \frac{\varphi^{(1)}\left[a ; b, b^{\prime} ; b b^{\prime} ; x, y\right]}{\varphi^{(1)}\left[a q ; b q, b^{\prime} q ; b b^{\prime} q^{2} ; x, y\right]} \\
& =\frac{T^{\prime}\left(a q, b q, b^{\prime} q\right)}{S^{\prime}\left(a, b, b^{\prime}\right)}+\frac{U^{\prime}\left(a q^{2}, b q^{2}, b^{\prime} q^{2}\right) / S^{\prime}\left(a, b, b^{\prime}\right)}{\frac{T^{\prime}\left(a q^{2}, b q^{2}, b^{\prime} q^{2}\right)}{S^{\prime}\left(a q, b q, b^{\prime} q\right)}+\frac{U^{\prime}\left(a q, b q, b^{\prime} q\right) / S^{\prime}\left(a q, b q, b^{\prime} q\right)}{\frac{\varphi^{(1)}\left[a q^{2} ; b q^{2}, b^{\prime} q^{2} ; b b^{\prime} q^{4} ; x, y\right]}{\varphi^{(1)}\left[a q^{3} ; b q^{3}, b^{\prime} q^{3} ; b b^{\prime} q^{6} ; x, y\right]}}+\cdots}
\end{aligned}
$$

$S^{\prime}, T^{\prime}$ and $U^{\prime}$ are defined in the proof.

Proof. By putting $q_{1}=q$ in our Theorem 1 [10, p.31] and after a little simplification, we have the Theorem 1 of Andrews [1, p.619]:

$$
\varphi^{(1)}\left[a ; b, b^{\prime} ; c, x, y\right]=\frac{(a)_{\infty}(b x)_{\infty}\left(b^{\prime} y\right)_{\infty}}{(c)_{\infty}(x)_{\infty}(y)_{\infty}}{ }_{3} \phi_{2}\left(\frac{c}{a} x, y ; b x, b^{\prime} y ; q, a\right) .
$$

Now taking $c=b b^{\prime}$ in (5.1) we have

$$
\varphi^{(1)}\left[a ; b, b^{\prime} ; b b^{\prime}, x, y\right]=\frac{(a)_{\infty}(b x)_{\infty}\left(b^{\prime} y\right)_{\infty}}{\left(b b^{\prime}\right)_{\infty}(x)_{\infty}(y)_{\infty}} \phi_{3}\left(\frac{b b^{\prime}}{a} x, y ; b x, b^{\prime} y ; q, a\right) .
$$


Making the change of the variables $a \rightarrow \frac{b b^{\prime}}{a}, b \rightarrow x, c \rightarrow y, d \rightarrow b x, e \rightarrow b^{\prime} y$, in the contiguous relation given by Andrews and Bowman $[3$, p.3347, (3.13)] we have the relation

$$
\begin{aligned}
S^{\prime}\left(a, b, b^{\prime}\right) \varphi^{(1)}\left[a ; b, b^{\prime} ; b b^{\prime} ; x, y\right]= & T^{\prime}\left(a q, b q, b^{\prime} q\right) \varphi^{(1)}\left[a q ; b q, b^{\prime} q ; b b^{\prime} q^{2} ; x, y\right] \\
& +U^{\prime}\left(a q^{2}, b q^{2}, b^{\prime} q^{2}\right) \varphi^{(1)}\left[a q^{2} ; b q^{2}, b^{\prime} q^{2} ; b b^{\prime} q^{4} ; x, y\right](5 .)
\end{aligned}
$$

where

$$
\begin{aligned}
S^{\prime}= & \frac{\left(b b^{\prime}\right)_{4}\left(1-b b^{\prime} q^{2}\right)}{a} \\
T^{\prime}= & \left(b b^{\prime} q\right)_{3}\left[\left(\frac{b b^{\prime}}{a}(x+y)+b x+b^{\prime} y\right)(1+q)-\left((x+y)+\frac{b x}{a}+\frac{b^{\prime} y}{a}\right)\right. \\
& \left.+\left(1+b b^{\prime} q\right)+\frac{\left(b b^{\prime} ; q^{2}\right)_{2}}{a}\right] \\
U^{\prime}= & (1-a q)\left(1-b b^{\prime}\right)\left(1-b b^{\prime} q / a\right)(1-b q)\left(1-b^{\prime} q\right)(y-b x q)\left(x-b^{\prime} y q\right) .
\end{aligned}
$$

Iterating the relation (5.3), we have the Theorem 2 .

\section{Summation formulae and continued fractions}

The following theorems give summation formulae for bibasic Appell function $\bar{\varphi}^{(1)}$. We also give continued fraction representation for these bibasic Appell series.

\section{Theorem 3.}

$$
\begin{aligned}
\bar{\varphi}^{(1)}\left[a ; b, b^{\prime} ; q^{k} ; b^{\prime} y, y ; q, q^{k}\right] & =\frac{\left(a q^{k} ; q^{k}\right)_{\infty}}{(1-y)\left(q^{k} ; q^{k}\right)_{\infty}}{ }_{2} \phi_{1}\left(\frac{q^{k}}{a}, y ; y q^{k} ; q^{k}, a\right) \\
& =\frac{\left(a y ; q^{k}\right)_{\infty}}{\left(y ; q^{k}\right)_{\infty}}
\end{aligned}
$$

Proof. Putting $x=b^{\prime} y, b b^{\prime}=y, c=q^{k}$ in (4.1) and summing the ${ }_{2} \phi_{1}$ by Heine's $q$ analogue of Gauss' summation formula $\underline{6}$, p.10, (1.5.1)], we have the summation Theorem 3 .

Theorem 4.
(i) $\bar{\varphi}^{(1)}\left[q^{2} ; b, b^{\prime} ; q^{5} ; b^{\prime} q^{2}, q^{2} ; q, q^{5}\right]=\frac{\left(q^{4} ; q^{5}\right)_{\infty}}{\left(q^{2} ; q^{5}\right)_{\infty}}$.

(ii) $\frac{\left(q ; q^{5}\right)_{\infty}}{\left(q^{3} ; q^{5}\right)_{\infty}} \bar{\varphi}^{(1)}\left[q^{2} ; b, b^{\prime} ; q^{5} ; b^{\prime} q^{2}, q^{2} ; q, q^{5}\right]=\frac{1}{1+\frac{q}{1+\frac{q^{2}}{1+\frac{q^{3}}{1+\cdots}}}}$ 


\section{Proof.}

(i) Putting $a=q^{2}, y=q^{2}, k=5$ in Theorem 3, we have the summation Theorem 4(i).

(ii) Ramanujan's celebrated $C(q)$ function

$$
C(q) \equiv \frac{1}{1+\frac{q}{1+\frac{q^{2}}{1+\frac{q^{3}}{1+\cdots}}}}=\frac{\left(q ; q^{5}\right)_{\infty}\left(q^{4} ; q^{5}\right)_{\infty}}{\left(q^{2} ; q^{5}\right)_{\infty}\left(q^{3} ; q^{5}\right)_{\infty}}
$$

Using Theorem 4(i), we have Theorem 4(ii). This gives a relation between bibasic Appell function $\bar{\varphi}^{(1)}$ and Ramanujan's function $C(q)$.

\section{Theorem 5 .}

$$
\begin{aligned}
& \text { Theorem 5. } \\
& \text { (i) } \bar{\varphi}^{(1)}\left[q^{2} ; b, b^{\prime} ; q^{6} ; b^{\prime} q^{3}, q^{3} ; q, q^{6}\right]=\frac{\left(q^{5} ; q^{6}\right)_{\infty}}{\left(q^{3} ; q^{6}\right)_{\infty}} . \\
& \text { (ii) } \frac{\left(q ; q^{6}\right)_{\infty}}{\left(q^{3} ; q^{6}\right)_{\infty}} \bar{\varphi}^{(1)}\left[q^{2} ; b, b^{\prime} ; q^{6} ; b^{\prime} q^{3}, q^{3} ; q, q^{6}\right]=\frac{1}{1+\frac{q+q^{2}}{1+\frac{q^{2}+q^{4}}{1+\frac{q^{3}+q^{6}}{1+\cdots}}}}
\end{aligned}
$$

\section{Proof.}

(i) Putting $a=q^{2}, y=q^{3}, k=6$ in Theorem 3, we have Theorem 5(i).

(ii) Using the relation 2, p.105, (7.12)] and Theorem 5(i), we have Theorem 5(ii). This gives a relation between bibasic Appell function $\bar{\varphi}^{(1)}$ and Ramanujan's continued fraction.

Theorem 6.

(i) $\bar{\varphi}^{(1)}\left[q^{4} ; b, b^{\prime} ; q^{8} ; b^{\prime} q^{3}, q^{3} ; q, q^{8}\right]=\frac{\left(q^{7} ; q^{8}\right)_{\infty}}{\left(q^{5} ; q^{8}\right)_{\infty}}$.

(ii) $\frac{\left(q ; q^{8}\right)_{\infty}}{\left(q^{3} ; q^{8}\right)_{\infty}} \bar{\varphi}^{(1)}\left[q^{4} ; b, b^{\prime} ; q^{8} ; b^{\prime} q^{3}, q^{3} ; q, q^{8}\right]=\frac{1}{1+\frac{q^{2}+q}{1+\frac{q^{4}}{1+\frac{q^{6}+q^{3}}{1+\cdots}}}}$

\section{Proof.}

(i) Putting $a=q^{4}, y=q^{3}, k=8$ in Theorem 3, we have Theorem 6(i).

(ii) Using the relation 2, p.105 (7.13)] and Theorem 6(i), we have Theorem 6(ii). This gives a relation between bibasic Appell function $\bar{\varphi}^{(1)}$ and Ramanujan's continued fraction. 


\section{Theorem 7 .}

$$
\begin{aligned}
& \frac{\left(q^{12} ; q^{20}\right)_{\infty}}{\left(q^{16} ; q^{20}\right)_{\infty}} \bar{\varphi}^{(1)}\left[q^{4} ; b, b^{\prime} ; q^{20} ; b^{\prime} q^{4}, q^{4} ; q, q^{20}\right] \\
& \quad=1+q-\frac{q}{1+q+q^{2}-\frac{q}{1+q+q^{3}-\frac{q}{1+q+q^{4}-\cdots}}} \\
& \quad=\frac{\left(q^{8} ; q^{20}\right)_{\infty}}{\left(q^{4} ; q^{20}\right)_{\infty}} \cdot \frac{\left.q^{12} ; q^{20}\right)_{\infty}}{\left(q^{16} ; q^{20}\right)_{\infty}}
\end{aligned}
$$

Proof. Putting $a=q^{4}, y=q^{4}, k=20$ in Theorem 3, and using the relation 5, Theorem 6, p.747], we have Theorem 7 .

The numerator of the continued fraction gives the remarkable complement to Euler's theorem that the partition of any integer $n>0$ into distinct parts are equinumerous with those of odd parts [5, Theorem 7, p.747].

\section{References}

[1] G. E. Andrews, Summations and transformations for basic Appell series, J. London Math. Soc. 4(1972), 618-622.

[2] G. E. Andrews, An introduction to Ramanujan's 'Lost' notebook, Amer. Math. Monthly 86(1979), 89-108.

[3] G. E. Andrews and Douglas Bowman, A full extension of the Rogers-Ramanujan continued fraction, Proc. Amer. Math. Soc. 123(1995), 3343-3350.

[4] P. Appell and J. Kampe De Feriet, Fonctions Hypergeometriques et Hyperspheriques, Gauthier villars, Paris, 1926.

[5] Basil Gordon, Some continued fractions of the Rogers-Ramanujan type, Duke Math. J. 32(1965), 741-748.

[6] G. Gasper and M. Rahman, Basic Hypergeometric Series, Cambridge University Press, Cambridge, 1990.

[7] F. H. Jackson, On basic double hypergeometric functions, Quart. J. Math. 13(1942), 69-82.

[8] F. H. Jackson, Basic double hypergeometric functions, Quart. J. Math. 15(1944), 49-61.

[9] L. J. Slater, Generalized Hypergeometric Functions, Cambridge University Press, Camgridge, 1966.

[10] Bhaskar Srivastava, A note on certain bibasic q-Appell and Lauricella series, Glasnik Matematicki, 30(50)(1955), 29-36.

Department of Mathematics, Lucknow University, Lucknow, India. 